\title{
A PROduÇão CIENTÍfica INTERnACIONAL SOBRE REDUÇÃo DE DANOS: UMA ANÁliSE COMPARATIVA ENTRE MEDLINE E LILACS ${ }^{1}$
}

\author{
Vilmar Ezequiel dos Santos²; \\ Cássia Baldini Soares ${ }^{3}$; Célia Maria Sivalli Campos ${ }^{4}$
}

O objetivo deste trabalho foi descrever e analisar o percurso da redução de danos (RD) nos países onde foi difundida. Partiu-se do pressuposto de que a RD significa uma nova forma de compreender e intervir nos problemas relacionados ao uso de drogas. O método consistiu na revisão das publicações científicas disponibilizadas nas bases MEDLINE, no período de 1996 a 2006 e LILACS, sem restrição de período. A análise das publicações aponta para difusão das estratégias e ampliação do campo da RD em vários países, e mostra diferenças significativas nas suas características, quando comparados países de capitalismo central e periférico.

Descritores: Redução do Dano; Saúde Pública; Drogas Ilícitas; Literatura de Revisão como Assunto.

\footnotetext{
${ }^{1}$ Artigo extraído da dissertação de mestrado "O objeto/sujeito da redução de danos: uma análise da produção científica da perspectiva da Saúde Coletiva" apresentada à Escola de Enfermagem, Universidade de São Paulo, SP, Brasil.

${ }^{2}$ Enfermeiro, Doutorando em Enfermagem, Escola de Enfermagem, Universidade de São Paulo, SP, Brasil. E-mail: vilmar@usp.br.

${ }^{3}$ Enfermeira, Livre Docente, Professor Associado, Escola de Enfermagem, Universidade de São Paulo, SP, Brasil. E-mail: cassiaso@usp.br.

${ }^{4}$ Enfermeira, Doutor em Enfermagem, Professor Doutor, Escola de Enfermagem, Universidade de São Paulo, SP, Brasil. E-mail: celiasiv@usp.br.
} 


\section{THE INTERNATIONAL SCIENTIFIC PRODUCTION ON HARM REDUCTION: A COMPARATIVE ANALYSIS BETWEEN MEDLINE AND LILACS}

The objective of this study was to describe and analyze the course of harm reduction (HR) in the countries where it was diffused. I started from the assumption that HR means new way of understanding and intervening in the problems related to drug use. The method consisted in the revision of scientific publications available in the MEDLINE, in the period of 1996 to 2006 and LILACS bases, without timeframe restriction. The analysis of the publications points to the diffusion of strategies and widening of the HR field in several countries, and shows significant differences in their characteristics when compared to countries of central and peripheral capitalism.

Descriptors: Reducción del Daño, Salud Pública, Drogas Ilícitas; Literatura de Revisión como Asunto.

\section{LA PRODUCCIÓN CIENTÍFICA INTERNACIONAL SOBRE REDUCCIÓN DE DAÑOS: UN ANÁLISIS COMPARATIVO ENTRE MEDLINE y LILACS}

El objetivo de este trabajo fue describir y analizar el trayecto de la reducción de daños (RD) en los países donde fue difundida. Se partió del presupuesto de que la RD significa una nueva manera de comprender e intervenir en los problemas relacionados al uso de drogas. El método consistió en la revisión de las publicaciones científicas hechas disponibles en las bases MEDLINE, en el período de 1996 a 2006 y LILACS, sin restricción de período. El análisis de las publicaciones apunta para difusión de las estrategias y ampliación del campo de la $\mathrm{RD}$ en diversos países, y muestra diferencias significativas en sus características, cuando comparados a países de capitalismo central y periférico.

Descriptores: Harm Reduction; Public Health; Street Drugs; Review Literature as Topic.

\section{Introdução}

A redução de danos $(\mathrm{RD})$, nas dimensões teórica e prática, consolidou-se como interface entre as respostas sociais à produção, ao comércio e ao consumo de drogas, e as respostas do campo da saúde pública ${ }^{(1)}$.

A origem da $\mathrm{RD}$, como prática voltada para o consumo de substâncias psicoativa, pode ser localizada na Inglaterra, em 1926, com o Relatório de Rolleston. Considerava-se que, mesmo diante de um consumo dependente de drogas, os usuários poderiam diminuir as consequências mais danosas, minimizando os efeitos prejudiciais à sua saúde ${ }^{(2)}$.

Foi na década de $80^{(3)}$, no entanto, que a RD passou a ser reconhecida como estratégia de saúde pública voltada a usuários de drogas, como uma das respostas à epidemia da AIDS. As políticas de saúde tornaram-se mais flexíveis e mais tolerantes com o usuário de drogas, incorporando estratégias para diminuir prejuízos relacionados ao consumo. A RD foi sendo incorporada pela saúde pública de vários países ${ }^{(4-6)}$, tomando as feições dos diferentes contextos sociais, políticos e econômicos, e difundindo-se como nova forma de compreender e intervir nos problemas relacionados ao consumo de drogas. Nessa trajetória, suscitou diversos questionamentos para o campo das políticas públicas.

Cabe salientar que os problemas relacionados ao consumo de drogas têm apresentado dimensões extraordinárias nas últimas décadas, exigindo respostas dos governos em âmbito nacional e internacional. A 
política adotada pela quase totalidade dos países vem, historicamente, enfatizando estratégias repressivas, punitivas e com foco voltado unicamente para a abstinência total de qualquer consumo de drogas, em especial as ilícitas.

Este estudo adotou os fundamentos do campo da saúde coletiva, para compreender que o consumo prejudicial de drogas tem suas raízes ligadas diretamente às formas de funcionamento da vida social, e que o complexo sistema de produção, comércio e consumo de drogas está diretamente relacionado ao sistema produtivo e econômico, não se referindo somente a processos individuais dos consumidores (liberdade de escolha, fragilidade individual etc.). Na formação social capitalista, a droga é percebida sob forma de mercadoria, potencializada pelas indústrias lícitas e ilícitas, para atender às necessidades de valorização do capital $^{(2)}$. Assim, para a saúde coletiva, a RD se configuraria como uma resposta mais coerente e humana de intervenção sobre os problemas oriundos da relação dos diferentes grupos sociais com as drogas.

Uma vez que a RD foi incorporada nas políticas voltadas ao consumo de drogas, em âmbito nacional e internacional, pretendeu-se, neste estudo, descrever e analisar as características dessa incorporação nos vários países onde foi adotada.

Assim, este estudo apresenta uma análise do processo de implementação da RD, a partir da produção científica disponível nas bases de dados MEDLINE e LILACS, caracterizando diferenças entre os países de capitalismo central e periférico, por meio de revisão suficientemente abrangente, para permitir a compreensão do percurso internacional da RD, embora não se caracterize como revisão sistemática exaustiva da literatura.

\section{Procedimentos metodológicos}

A revisão bibliográfica foi realizada em julho de 2006, enfocando-se os dados da MEDLINE, de 1996 a 2006 e, sem definição de período, os dados da LILACS. Não se utilizou o descritor "redução de danos" por não ser reconhecido pela BIREME na ocasião da revisão bibliográfica. Assim, para ampliar ao máximo a captação de trabalhos sobre o tema e, por outro lado, limitá-la aos trabalhos que versavam sobre drogas psicoativas, lícitas e ilícitas, foram utilizadas combinações de descritores reconhecidos. Na base MEDLINE utilizaram-se: "redução do dano" e "transtornos relacionados ao uso de substâncias psicoativas" ou "drogas ilícitas" ou "alcoolismo" ou "tabagismo"; e na base LILACS, "redução" ou "danos" e "transtornos relacionados ao uso de substâncias psicoativas" ou "drogas ilícitas" ou "redução do dano" ou "alcoolismo" ou "tabagismo".

Cabe ressaltar que o descritor "redução de danos" (harm reduction) foi introduzido no MeSH (Medical Subject Headings), um vocabulário controlado da National Library of Medicine, somente em 2003, o que se mostrou como um fator de dificuldade para a localização de trabalhos no MEDLINE, em anos anteriores.

Primeiramente procedeu-se à leitura de todos os resumos dos trabalhos encontrados nas duas bases de dados para, em seguida, selecionar os que tratavam diretamente da teoria e/ou prática da RD relacionada ao campo das drogas psicoativas lícitas e/ou ilícitas. Os trabalhos selecionados foram lidos na íntegra, o que possibilitou a formulação de um sistema de classificação das várias formas de apresentação da RD nos distintos países e localidades.

\section{Resultados}

Na Tabela 1 apresenta-se uma comparação entre os países de origem dos trabalhos, indicando que a quase totalidade dos trabalhos encontrados na MEDLINE era proveniente de países de capitalismo central (92\%), e a quase totalidade dos encontrados na base LILACS era de países de capitalismo periférico (98\%). A distribuição dos trabalhos apresentados na Tabela 1 é coerente com a proporcionalidade das revistas indexadas na base MEDLINE, que é mantida pela U.S.National Library of Medicine, na qual menos de 10\% dos títulos indexados são originários de países em desenvolvimento. Quase 70\% dos 4.800 títulos são publicados em países de língua inglesa e, entre esses, $90 \%$ concentram-se no hemisfério norte, $44 \%$ dos quais estão nos $\operatorname{EUA}^{(7)}$. Já na base LILACS, encontram-se 605 revistas indexadas, da área da saúde, publicadas nos países da América Latina e do Caribe.

Tabela 1 - País de origem das publicações referentes à revisão bibliográfica sobre $\mathrm{RD}$, realizada na MEDLINE e na LILACS. São Paulo, SP, Brasil, 2006

\begin{tabular}{|c|c|c|c|c|}
\hline \multirow{2}{*}{ País } & \multicolumn{2}{|c|}{ MEDLINE } & \multicolumn{2}{|c|}{ LILACS } \\
\hline & $\mathbf{n}$ & $\%$ & $\mathbf{n}$ & $\%$ \\
\hline América do Norte: Estados Unidos & 48 & 55 & 0 & 0 \\
\hline América do Norte: Canadá & 9 & 11 & 0 & 0 \\
\hline Oceania: Austrália & 7 & 8 & 0 & 0 \\
\hline Reino Unido: Inglaterra e País de Gales & 8 & 9 & 0 & 0 \\
\hline $\begin{array}{l}\text { Europa: França, Espanha, Holanda, } \\
\text { Alemanha e Suécia }\end{array}$ & 8 & 9 & 1 & 2 \\
\hline América Latina: Brasil & 2 & 2 & 41 & 87 \\
\hline América Latina: Argentina, Chile, Colômbia & 0 & 0 & 5 & 11 \\
\hline Informação não localizada & 5 & 6 & 0 & 0 \\
\hline Total & 87 & 100 & 47 & 100 \\
\hline
\end{tabular}

$\mathrm{Na}$ MEDLINE, somente foram encontradas publicações a respeito de RD a partir do ano 2002. $\mathrm{Na}$ LILACS, elas datam de 1994. Pode-se inferir que, nos países de capitalismo periférico, a preocupação com a divulgação da RD foi anterior, em função das características da epidemia da AIDS e da mobilização social, que pressionaram o poder público a tomar o problema como objeto de políticas governamentais de saúde pública. Reitera essa afirmação a significativa inserção institucional dos autores.

Dos artigos da LILACS, $41 \%$ eram vinculados a órgãos governamentais da área da saúde (26\% a ministérios e $15 \%$ a secretarias); $46 \%$ eram vinculados a universidades e $13 \%$ a organizações não governamentais (ONG). 
Já na MEDLINE, 60\% dos autores eram vinculados a universidades, $12 \%$ a serviços de atenção à saúde $11 \%$ a agências e institutos, $2 \%$ a ONGs, $2 \%$ a empresas privadas e $2 \%$ a jornais. Em $10 \%$ dos artigos não constava essa informação.

A partir dos dados apresentados na Tabela 2, constatase que, na MEDLINE, a maioria dos artigos (78\%) tinha centralidade na droga - a substância, em particular, ou a dependência dela. Com proporcionalidade diversa, na LILACS, em apenas $4 \%$ dos artigos, a droga era tema central. Ainda na LILACS, uma parcela considerável dos trabalhos (38\%) versava sobre AIDS e avaliação de programas, sendo que na MEDLINE esse percentual era de 4\%. Em relação a aspectos teóricos e políticos, as diferenças também são importantes, indo de $43 \%$ na LILACS para $15 \%$ na MEDLINE. Outro dado que chama a atenção é que, apenas na LILACS, foram encontrados trabalhos que tratavam de prevenção ao consumo prejudicial de drogas.

Tabela 2 - Temas principais comparados entre trabalhos da MEDLINE e da LILACS. São Paulo, SP, Brasil, 2006.

\begin{tabular}{lcccc}
\hline \multirow{2}{*}{ Tema principal } & \multicolumn{2}{c}{ LILACS } & \multicolumn{2}{c}{ MEDLINE } \\
\cline { 2 - 5 } & $\mathbf{n}$ & $\%$ & $\mathbf{n}$ & $\%$ \\
Tabaco & 0 & 0 & 39 & 45 \\
Tratamento de dependência & 0 & 0 & 17 & 20 \\
Álcool & 1 & 2 & 10 & 12 \\
Aspectos teóricos e éticos & 12 & 26 & 9 & 10 \\
Políticas & 8 & 17 & 4 & 5 \\
Drogas em festas & 0 & 0 & 3 & 3 \\
Avaliação de programas & 7 & 15 & 3 & 3 \\
Crack & 1 & 2 & 1 & 1 \\
Doenças Transmissíveis & 11 & 23 & 1 & 1 \\
Prevenção & 7 & 15 & 0 & 0 \\
Total & 47 & 100 & 87 & 100 \\
\hline
\end{tabular}

A Tabela 3 apresenta uma classificação dos trabalhos encontrados na MEDLINE e na LILACS, por temas e subtemas relacionados à RD. Para oferecer maior riqueza de conteúdo na classificação, optou-se por incluir alguns trabalhos em mais de um subtema.

Tabela 3 - Distribuição por tema central, subtemas na MEDLINE e LILACS. São Paulo, SP, Brasil, 2006.

\begin{tabular}{clcc}
\hline $\begin{array}{c}\text { Tema } \\
\text { principal }\end{array}$ & \multicolumn{1}{c}{ Subtema } & MEDLINE & LILACS \\
\hline Tabaco & $\begin{array}{l}\text { Diminuição dos níveis de } \\
\text { toxicidade dos elementos } \\
\text { cancerígenos }\end{array}$ & 19 & 0 \\
& $\begin{array}{l}\text { Formas alternativas de tratamento } \\
\text { para diminuir ou cessar o } \\
\text { consumo }\end{array}$ & 13 & 0 \\
$\begin{array}{l}\text { Percepção da população sobre o } \\
\text { impacto das políticas }\end{array}$ & 3 & 0 \\
& $\begin{array}{l}\text { Aspectos teóricos e éticos } \\
\text { Políticas de RD para o tabaco }\end{array}$ & 3 & 0 \\
& & 1 & 0
\end{tabular}

(continua...)
Tabela 3 - continuação

\begin{tabular}{|c|c|c|c|}
\hline $\begin{array}{c}\text { Tema } \\
\text { principal }\end{array}$ & Subtema & MEDLINE & LILACS \\
\hline \multirow[t]{4}{*}{ Álcool } & Tratamento e uso moderado & 4 & 0 \\
\hline & $\begin{array}{l}\text { Consumo de álcool entre } \\
\text { estudantes universitários }\end{array}$ & 2 & 0 \\
\hline & Cuidado na atenção primária & 1 & 0 \\
\hline & Políticas sobre o álcool & 3 & 1 \\
\hline Crack & $\begin{array}{l}\text { Fornecimento de kits para o uso } \\
\text { mais seguro }\end{array}$ & 1 & 1 \\
\hline \multirow[t]{3}{*}{ Tratamento } & $\begin{array}{l}\text { Estratégia complementar ao } \\
\text { tratamento tradicional }\end{array}$ & 13 & 0 \\
\hline & $\begin{array}{l}\text { Acesso a populações em situação } \\
\text { de rua }\end{array}$ & 3 & 0 \\
\hline & Terapias de substituição & 1 & 0 \\
\hline \multirow{4}{*}{$\begin{array}{l}\text { Aspectos } \\
\text { teóricos e } \\
\text { éticos }\end{array}$} & $\begin{array}{l}\text { Princípios gerais da RD e } \\
\text { estratégias de intervenção }\end{array}$ & 4 & 5 \\
\hline & $\begin{array}{l}\text { Programas oficiais e participação } \\
\text { social }\end{array}$ & 0 & 7 \\
\hline & $\begin{array}{l}\text { Conflitos vivenciados por } \\
\text { profissionais }\end{array}$ & 4 & 0 \\
\hline & Ética em pesquisas & 1 & 0 \\
\hline \multirow[t]{4}{*}{$\begin{array}{l}\text { Drogas em } \\
\text { festas }\end{array}$} & $\begin{array}{l}\text { Iniciativas associadas à segurança } \\
\text { básica }\end{array}$ & 1 & 0 \\
\hline & $\begin{array}{l}\text { Percepção de riscos e benefícios } \\
\text { do consumo }\end{array}$ & 1 & 0 \\
\hline & Utilização de drogas em clubes & 1 & 0 \\
\hline & $\begin{array}{l}\text { Modificações ambientais como } \\
\text { forma de RD }\end{array}$ & 1 & 0 \\
\hline AIDS & $\begin{array}{l}\text { Prevenção de doenças } \\
\text { transmissíveis entre usuários de } \\
\text { droga injetável (UDI) }\end{array}$ & 0 & 10 \\
\hline \multirow{2}{*}{$\begin{array}{l}\text { Avaliação } \\
\text { de } \\
\text { programas }\end{array}$} & $\begin{array}{l}\text { Impactos positivos na diminuição } \\
\text { da transmissão do HIV }\end{array}$ & 1 & 7 \\
\hline & $\begin{array}{l}\text { Aceitação de diferentes } \\
\text { estratégias de RD por diferentes } \\
\text { países }\end{array}$ & 2 & 0 \\
\hline \multirow[t]{7}{*}{ Políticas } & $\begin{array}{l}\text { Aumento do preço e entrada de } \\
\text { novas drogas no mercado }\end{array}$ & 1 & 0 \\
\hline & $\begin{array}{l}\text { Mudanças e incorporação da RD } \\
\text { na orientação das políticas }\end{array}$ & 0 & 8 \\
\hline & $\begin{array}{l}\text { Consequências da política para os } \\
\text { países produtores }\end{array}$ & 0 & 1 \\
\hline & $\begin{array}{l}\text { Aceitação e percepção da RD pela } \\
\text { população }\end{array}$ & 5 & 0 \\
\hline & $\begin{array}{l}\text { Panorama da RD nas políticas } \\
\text { internacionais }\end{array}$ & 0 & 3 \\
\hline & Adoção da RD em prisões & 1 & 0 \\
\hline & $\begin{array}{l}\text { Ações voltadas à prevenção da } \\
\text { AIDS }\end{array}$ & 1 & 0 \\
\hline Prevenção & $\begin{array}{l}\text { Modelos de educação em RD para } \\
\text { prevenção do consumo prejudicial } \\
\text { de drogas }\end{array}$ & 0 & 7 \\
\hline
\end{tabular}

\section{Discussão}

Quanto à abordagem de aspectos teóricos, as publicações da MEDLINE apresentavam princípios gerais da RD e estratégias de intervenção, inclusive em clubes noturnos e raves; e os de aspectos éticos enfocavam conflitos profissionais no atendimento de pessoas com uso abusivo de drogas, ou aspectos da ética em pesquisas.

Já na LILACS, nos estudos que abordavam aspectos teóricos, especialmente nos brasileiros, os autores eram ligados a organizações governamentais e ao governo federal e tratavam de conceitos, aspectos legais e éticos, aspectos 
da implementação de programas de RD como estratégia de saúde pública. Os que abordavam a RD na saúde pública visavam a prevenção de doenças transmissíveis em usuários de droga injetável (UDI), perspectiva do início da implantação da RD no Brasil, unicamente para prevenir doenças transmissíveis em UDI, sem visar mudanças no padrão de uso de drogas. Cabe ressaltar que a Organização Mundial da Saúde (OMS) tem apoiado países que adotaram as estratégias de $\mathrm{RD}$, incentivando tanto as práticas de prevenção da disseminação do $\operatorname{HIV}_{-} \operatorname{AIDS}^{(8)}$ quanto as práticas mais direcionadas a consumidores de drogas que não frequentam os serviços tradicionais de atendimento a dependentes, bem como as terapias voltadas à troca do consumo de substâncias mais danosas por outras menos danosas à saúde.

A análise dos artigos da LILACS indicou tendências das políticas voltadas ao consumo de drogas em países de capitalismo periférico. Observou-se, nos artigos dessa base, maior inserção da RD em políticas oficiais, bem como a utilização de referenciais críticos a elas, por considerarem as diferenças regionais e as desigualdades sociais entre os países.

Artigos de autores brasileiros apresentavam a RD para mudanças na orientação das políticas, apontavam determinantes estruturais do consumo prejudicial de drogas na contemporaneidade, e propunham iniciativas para a formação de sujeitos críticos e conscientes das várias dimensões do consumo de drogas. Segundo alguns autores $^{(1)}$, a droga é concebida como mercadoria, e esses propõem que "o consumo de drogas deve ser analisado à luz da estrutura e dinâmicas do modo de produção capitalista, que conformam os contextos da sociedade contemporânea".

Foi também maior o número de trabalhos na LILACS que discutiam políticas públicas e a incorporação de princípios da RD nas políticas voltadas ao consumo de drogas e, consequentemente, na operacionalização de programas vinculados a essas políticas. Havia, ainda, artigos que abordavam o panorama da RD nas políticas internacionais - um deles discutia criticamente os princípios da política de guerra às drogas ${ }^{(9)}$. Alguns artigos discutiam a incorporação da RD nas políticas públicas brasileiras.

A porcentagem de trabalhos que apresentavam avaliação de programas de RD na LILACS foi bem maior do que na MEDLINE (15 e 3\%, respectivamente), ainda que os programas brasileiros apresentados fossem restritos a usuários de drogas injetáveis.

$\mathrm{Na}$ MEDLINE, não foram encontrados artigos voltados à prevenção. $\mathrm{Na}$ LILACS, encontrou-se, apenas em trabalhos brasileiros, referência a modelos de educação, como estratégia de RD para prevenção do consumo prejudicial de drogas para jovens ${ }^{(1,10-15)}$.

Os artigos da MEDLINE com centralidade em políticas de RD a serem adotadas pelo Estado - à exceção das políticas dirigidas ao álcool e tabaco - tratavam do controle do suprimento de drogas, do aumento de preço, e da entrada de novas drogas no mercado, ou de ações voltadas a indivíduos sob a custódia do Estado: os que estavam em prisões; ou, ainda, abordavam ações voltadas à prevenção da AIDS. Alguns artigos apresentavam resultados de pesquisas que examinavam a aceitação, pela população, da incorporação da RD nas políticas públicas.

Observou-se, no âmbito da discussão oficial da política internacional, a RD é tolerada, quando introduzida como estratégia submetida à política hegemônica de guerra às drogas, a fim de ser utilizada como última alternativa, para dependentes de drogas considerados refratários a qualquer tipo de intervenção voltada à abstinência da droga.

Coerentemente com a menor proporcionalidade de artigos da MEDLINE dedicados à discussão teórica da $\mathrm{RD}$ e de artigos com centralidade nas políticas públicas, foi também menor a proporção de trabalhos dedicados à implantação e avaliação de programas de RD. Encontrou-se um artigo, de origem francesa, que avaliava um programa centrado na utilização de estratégias para a diminuição da transmissão do HIV (distribuição de seringas e tratamentos de substituição de drogas injetáveis) ${ }^{(16)}$. Os demais artigos, de origem americana, examinavam a tendência à aceitação de estratégias de RD (distribuição de seringas, terapias de substituição, lugares seguros para consumo de drogas), em países europeus e nos Estados Unidos.

Ressalte-se, também, diferença marcante entre a proporcionalidade de artigos que traziam a associação entre RD e doenças transmissíveis (AIDS e hepatite), nas duas bases de dados. Na LILACS, essa associação era abordada em $23 \%$ dos artigos e, na MEDLINE, em apenas $2 \%$ deles. A avaliação de programas de RD, na LILACS, foi feita em $15 \%$ dos artigos, enquanto, na MEDLINE, em apenas $1 \%$ deles.

Vale reiterar a observação de que artigos da MEDLINE que abordavam a incorporação da RD nas políticas públicas, mesmo que restrita a estratégias pontuais e específicas, mostraram indícios de que a RD tem sido ainda pouco incorporada em países de capitalismo central. Nota-se que há preocupação maior em aquilatar a opinião, ou a aceitação, da população, a respeito da incorporação da RD nas políticas públicas, do que a preocupação em avaliar os impactos das ações advindas dessas políticas nos indivíduos e grupos sociais.

$\mathrm{Na}$ MEDLINE, como descrito anteriormente, grande parte dos artigos focalizava uma droga em particular (57\%), majoritariamente drogas lícitas. Desses, 78\% focalizavam o tabaco, 20\% o álcool e, apenas 2\%, drogas ilícitas, como o crack.

Dentre os estudos relacionados à RD voltada ao tabaco, a maioria era originária dos Estados Unidos (69\%) e abordava questões relacionadas à diminuição dos níveis de toxicidade dos elementos cancerígenos do cigarro; à regulação e controle, pelo Estado, da produção, comércio e consumo do cigarro; ao tratamento do dependente de tabaco; a políticas de RD voltadas ao uso de tabaco; à percepção da população do impacto das políticas de RD no consumo de tabaco, bem como reflexões e aspectos éticos do uso do tabaco.

Os artigos que tinham como foco a RD relacionada ao álcool abordavam-na como parte do tratamento da dependência, mas, também, como estratégia para diminuir 
prejuízos do consumo de álcool, inclusive entre estudantes, e como estratégia utilizada na atenção primária. Havia ainda artigos relacionados a políticas de $\mathrm{RD}$ voltadas ao álcool.

Os que focalizavam o tratamento da dependência de drogas tomavam a RD como estratégia complementar a ser utilizada como parte do tratamento convencional do dependente da droga, ou como estratégia para alcançar populações que não acessam os serviços de saúde para tratamento.

O único artigo com centralidade em droga ilícita - $\operatorname{crack}^{(17)}$ - ,era originário do Canadá, e propunha o fornecimento de kits para dependentes que não acessavam os serviços tradicionais de atenção à saúde.

Enquanto a maioria (57\%) dos artigos da MEDLINE tinha como foco as drogas lícitas (tabaco e álcool), na LILACS não foi encontrado nenhum artigo sobre $\mathrm{RD}$ relacionada ao tabaco, e apenas $2 \%$ tinham como preocupação o álcool ${ }^{(18)}$. Esses artigos discutiam o consumo prejudicial de álcool e suas consequências para o indivíduo, família e sociedade e estratégias da RD para minimizar prejuízos sociais. Havia apenas um artigo (2\%) na LILACS que abordava droga ilícita - $\operatorname{crack}^{(19)}$.

Observou-se, a partir deste estudo, certa homogeneidade na concepção de RD, quando tomada como sinônimo de estratégia, de práticas e intervenções relacionadas ao indivíduo ou ao ambiente, para diminuir comportamentos de risco à saúde (acidentes de trânsito, prevenção da AIDS, hepatites, problemas advindos do uso de drogas sintéticas), para diminuir prejuízos a indivíduos considerados refratários aos tratamentos e intervenções convencionais, para diminuição e substituição de substâncias, e para evitar overdoses e outros agravos à saúde. Esses trabalhos, que tomavam a RD sob essa perspectiva instrumental, não apresentavam discordâncias significativas, mesmo sendo advindos de países e continentes distintos, captados pelas duas bases de dados estudadas.

No entanto, quando se tratava de discussões de políticas e de orientação teórica, a RD se apresentava de maneira muito diversa. Nessa perspectiva, a RD era abordada como um apêndice da política hegemônica de guerra às drogas, apresentada como estratégia de prevenção terciária para populações vulneráveis, excluídas das alternativas de tratamentos tradicionais voltados à abstinência, majoritariamente de drogas lícitas. Essa abordagem era a preponderante nos trabalhos captados pela MEDLINE, ou seja, publicados em revistas de países de capitalismo central, a maioria delas dos EUA, país que, historicamente, tem sido governado por partidos que imprimem a lógica do mercado privado de cunho liberal - com pouca interferência do Estado na formulação e operacionalização das políticas sociais, de caráter eminentemente focalizado na população comprovadamente pobre ${ }^{(20)}$. Os países classificados como liberais têm, à exceção do Canadá e Grã-Bretanha, depois dos países ex-fascistas, os mais baixos investimentos públicos em cuidados à saúde, e a mais baixa cobertura de sistema público de atenção à saúde. Pode-se inferir que a Organização Panamericana de Saúde (OPS), ainda que não publicite críticas às políticas oficiais no campo das drogas, estimula que haja mudança de paradigma na relação entre profissionais de saúde e usuários, quando adota a concepção de RD como estratégia de saúde pública preventiva, que promove maior integração entre os usuários e os serviços de saúde, respeitando aspectos éticos e os direitos humanos, priorizando a manutenção da vida, e permitindo a redução das infecções e outros agravos à saúde ${ }^{(21)}$.

Outra perspectiva encontrada, exclusivamente nas discussões teóricas de artigos da base LILACS, foi a concepção da RD como paradigma em construção, que faz a crítica à política oficial de guerra às drogas e vai além da proposição de estratégias, reconhece o usuário de drogas ilícitas como sujeito de direitos, busca articulação com outros campos do saber - notadamente a educação e a ética - na tentativa de abarcar a complexidade do fenômeno contemporâneo do consumo de drogas.

Esse movimento encontrou eco nas políticas sociais públicas, especialmente no Brasil, que vem investindo na ampliação de ações de atenção ao usuário, e na prevenção do consumo prejudicial de drogas e, coerentemente, na avaliação de programas.

\section{Conclusão}

A revisão bibliográfica aqui empreendida mostrou significativas variações nas concepções e na implementação de ações de RD, entre os países de capitalismo central e os de capitalismo periférico. Enquanto nos primeiros a RD tem sido, com algumas exceções, considerada como uma estratégia de cunho operacional e de política instrumental, nos países de capitalismo periférico, principalmente no Brasil, ela vem tomando contorno mais amplo, no que diz respeito à renovação das políticas públicas, no campo das drogas.

$\mathrm{Na}$ presente revisão de literatura empreendida neste estudo, houve evidências de que a RD avançou, nas discussões teórica e ética, mais em países de capitalismo periférico do que em países de capitalismo central, o que favoreceu a incorporação dos seus conceitos nas políticas públicas voltadas a usuários de drogas, bem como a implantação de estratégias voltadas para a prevenção do consumo prejudicial de drogas, não se restringindo ao tratamento da dependência e a estratégias de acesso a populações excluídas.

\section{Referencias}

1. Canoletti B, Soares CB. Programas de prevenção ao consumo de drogas no Brasil: uma análise da produção científica de 1991 a 2001. Interface - Comunic Saúde Educ. 2005;9(16):115-29.

2. Santos VE, Soares CB, Campos CMV. Redução de danos: análise das concepções que orientam as práticas no Brasil. Physis Rev Saúde Colet. 2010;20(3):995-1015.

3. Stimsom GV. AAids e o uso de drogas injetáveis no Reino Unido, 1987-1993: As políticas públicas e a prevenção 
da epidemia. In: Bastos FI, Mesquita F, Marques LF, organizadores. Troca de seringas: ciência, debate e saúde pública. Brasília: Ministério da Saúde; 1998. p. 9-54.

4. Berridge V. Harm minimisation and public health: an historical perspective. In: Heather N, Wodak A, Nadelmann E, O'Hare P, editors. Psychoactive drugs and harm reduction: From faith to science. London: Whurr; 1993. p. 55-64.

5. Reale D. O caminho da redução de danos associados ao uso de drogas: do estigma a solidariedade. [Dissertação Mestrado em Medicina Preventiva]. São Paulo: Faculdade de Medicina, Universidade de São Paulo; 1997.

6. Fonseca EM. Políticas de redução de danos ao uso de drogas: o contexto internacional e uma análise preliminar dos programas brasileiros. [Dissertação Mestrado em Saúde Pública]. Rio de Janeiro: Escola Nacional de Saúde Pública Sergio Arouca, Fundação Oswaldo Cruz; 2005. $112 \mathrm{p}$.

7. Kotzin S. Journal selection for Medline. In: 71th World Library and Information Congress: IFLA General Conference and Council "Libraries - A voyage of discovery"; 2005 Aug 4th - 18th; Oslo, Norway. [evento na Internet]. Oslo; 2005. [acesso 16 jun 2011]. Disponível em: http://www.ifla.org/IV/ifla71/Programme.htm.

8. ONUSIDA/OMS. Programa Conjunto de las Naciones Unidas sobre el VIH/SIDA. Situación de la epidemia de SIDA. Ginebra: Organización Mundial de la Salud (OMS); 2005. (versión española, diciembre de 2005). p. 11.

9. Vargas Meza R. Fumigación y Conflicto: Políticas antidrogas y deslegitimación del Estado en Colombia. Santafé de Bogotá (CO): Tercer Mundo Editores; 1999.

10. Soares CB, Campos CMS. A responsabilidade da universidade pública no ensino da prevenção do uso prejudicial de drogas. Mundo Saúde. 2004;28(1):110-5.

11. Soares CB. Adolescentes, drogas e AIDS em São Paulo. Saúde Deb. 1995;46:44-53.

12. Veloso L, Carvalho J, Santiago L. Redução dos danos decorrentes do uso de drogas: uma proposta educativa no âmbito das políticas públicas. In: Bravo MIS, Vasconcelos AM, Gama AS, Monnerat GL. Saúde e serviço social. Rio de Janeiro: Cortez; 2004. p. 165-78.

13. Figueiredo RMMD, organizadora. Prevenção ao abuso de drogas em ações de saúde e educação: uma abordagem sócio-cultural e de redução de danos [Internet]. São Paulo: NEPAIDS-USP; 2002. [acesso 31 mar 2008]. Disponível em: http://www.usp.br/nepaids/drogas-as.pdf

14. Tavares-de-Lima FB. Prevenção ao uso de drogas: modelos utilizados na educação, suas relações e possibilidades quanto a atitudes preventivas [dissertação]. São Paulo: Departamento de Educação, Pontifícia Universidade Católica; 2003.

15. Ferreira VRT. Relato de uma experiência com adolescentes sobre o uso de drogas. Psicol Teor Prat. 2003;5(2):47-54.

16. Des Jarlais DC. Evaluating national harm reduction programs. Addiction. 2005;100(11):1575-6.

17. Haydon E, Fischer B. Crack use as a public health problem in Canada: call for an evaluation of safer crack use kits'. Can J Public Health. 2005;96(3):185-8.

18. Melcop AG, Maia D, Buning E, Franch M. Relatório da I Conferência Internacional sobre Consumo de Álcool e Redução de Danos: em busca de uma política abrangente para os países em transição e desenvolvimento. Recife: Rede Brasileira de Redução de Danos; 2003. 85 p.

19. Fochi EL, Moraes MS, Chiaravalloti F Neto, Gandolfi D, Ferreira EMA. Caracterização de 46 usuários de crack abordados pelo Programa de Redução de Danos "Tá Limpo". HB Cient. 2000;7(2):85-91.

20. Navarro V, Muntaner C, Borrell C, Benach J, Quiroga A, Rodriguez-Sanz M, Vergés N, Pasarín MI. Politics and health outcomes. Lancet. 2006;368:1033-7.

21. OPS. Organización Panamericana de la Salud. Manual para la atención y manejo integral de los usuarios de drogas viviendo con VIH/SIDA en América Latina y el Caribe. Washington, D.C: OPS, 2006.

\section{Como citar este artigo:}

Santos VE, Soares CB, Campos CMS. A produção científica internacional sobre redução de danos: Uma análise comparativa entre MEDLINE e LILACS. SMAD, Rev. Eletrônica Saúde Mental Álcool Drog. (Ed. port.). jan.-abr. 2012 [acesso: ];8(1):41-7. Disponível em:

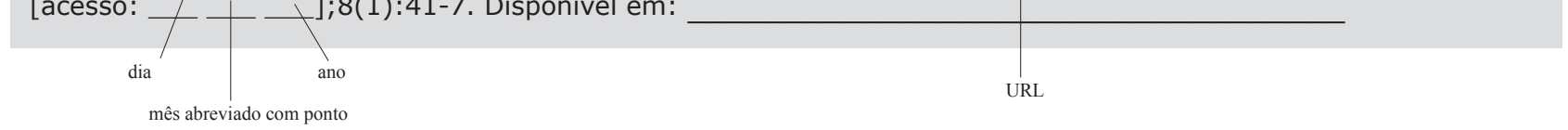

